\title{
New Baade-Wesselink distances and radii for four metal-rich Galactic Cepheids ${ }^{\star}$
}

\author{
S. Pedicelli ${ }^{1,2}$, B. Lemasle ${ }^{3}$, M. Groenewegen ${ }^{4}$, M. Romaniello ${ }^{1}$, G. Bono ${ }^{2,5}$, C. D. Laney ${ }^{6}$, P. François ${ }^{7}$

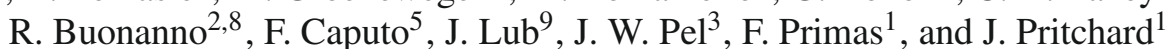 \\ ${ }^{1}$ European Southern Observatory (ESO), Karl-Schwarzschild-Strasse 2, 85748 Garching bei München, Germany \\ e-mail: spedicel@eso.org \\ 2 Università di Roma Tor Vergata, via della Ricerca Scientifica 1, 00133 Roma, Italy \\ 3 Kapteyn Institute, University of Groningen, PO Box 800, 9700 AV Groningen, The Netherlands \\ 4 Royal Observatory of Belgium, Ringlaan 3, 1180 Brussels, Belgium \\ INAF - Osservatorio Astronomico di Roma, via Frascati 33, Monte Porzio Catone, Italy \\ ${ }^{6}$ South African Astronomical Observatory, PO Box 9, 7935 Observatory, South Africa \\ 7 Observatoire de Paris-Meudon, GEPI, 61 avenue de l'Observatoire, 75014 Paris, France \\ 8 ASI-Science Data Center, ASDC c/o ESRIN, via G. Galilei, 00044 Frascati, Italy \\ 9 Leiden Observatory, Leiden University, PO Box 9513, 2300 RA Leiden, The Netherlands
}

Received 15 February 2010 / Accepted 12 March 2010

\section{ABSTRACT}

\begin{abstract}
Aims. We provide accurate estimates of distances, radii, and iron abundances of four metal-rich Cepheids, namely V340 Ara, UZ Sct, AV Sgr, and VY Sgr. The main aim of this investigation is to constrain their pulsation properties and their location across the Galactic inner disk.

Methods. We adopted new accurate NIR $(J, H, K)$ light curves and new radial velocity measurements for the target Cepheids to determinate their distances and radii using the Baade-Wesselink technique. In particular, we adopted the most recent calibrations of both the IR surface brightness relation and the projection factor. We also provide accurate measurements of the iron abundance of the target Cepheids.

Results. Current distance estimates agree to within one $\sigma$ with similar distances based on either empirical or theoretical NIR PeriodLuminosity (P-L) relations. However, the uncertainties in the Baade-Wesselink distances are on average a factor of 3-4 smaller than errors affecting other distance determinations. Mean Baade-Wesselink radii also agree at the one $\sigma$ level with Cepheid radii based either on empirical or theoretical Period-Radius relations. Iron abundances are, within one $\sigma$, similar to those determined by Andrievsky and collaborators, thus confirming that the target Cepheids are super metal-rich. We also found that the luminosity amplitudes of classical Cepheids, at odds with RR Lyrae stars, do not exhibit a clear correlation with their metal content. This circumstantial evidence appears to be caused by the Hertzsprung progression and the dependence of the topology of the instability strip on metallicity, evolutionary effects, and binaries.
\end{abstract}

Key words. stars: abundances - stars: distances - stars: variables: Cepheids - Galaxy: disk - stars: oscillations

\section{Introduction}

Classical Cepheids are used as both standard candles and tracers of young stellar populations (Maciel \& Costa 2009; Pedicelli et al. 2009). They are bright and variable objects and thanks to the Hubble Space Telescope they have been identified and accurately measured in both Local Group $(d \lesssim 1 \mathrm{Mpc})$ and Local Volume $(d \lesssim 10 \mathrm{Mpc}$ ) galaxies (Freedman et al. 2001; Tammann et al. 2003; Bono et al. 2008). They obey a PeriodLuminosity (PL) relation and are the most popular primary distance indicators (Feast 1999; Macri et al. 2006; Fouqué et al. 2007; Groenewegen 2007; di Benedetto 2008; Groenewegen 2008; Kervella et al. 2008; Kanbur et al. 2009; Marengo et al. 2009; Scowcroft et al. 2009). In spite of these considerable observational and theoretical efforts (Marconi 2009, and references therein), the universality of the optical and nearinfrared (NIR) PL relations has not been empirically verified

* Based on observations made with MPG/ESO $2.2 \mathrm{~m}$ telescope at La Silla Observatory under proposal IDs: 75.D-0676, 60.A-9120 and multi-epoch, multi-band NIR data at SAAO.
(Benedict et al. 2007; Romaniello et al. 2008; Sandage et al. 2009), even though the zero-point and the slope of the PL relation have been estimated with a variety of independent methods.

The calibration and the validation of the PL relation should be based on distances measured with a geometrical method such as trigonometric parallaxes. This approach was adopted by Benedict et al. (2007), who measured parallaxes with a mean accuracy of $8 \%$ for nine Galactic Cepheids using the fine guidance sensor (FGS) on board the Hubble Space Telescope (HST). A new revision of HIPPARCOS parallaxes for Galactic Cepheids (244 objects) was provided by van Leeuwen (2007) and van Leeuwen et al. (2007). The accuracy of the new measurements is on average a factor of two higher than the old ones. However, the Cepheids with the most accurate HIPPARCOS parallaxes are Polaris ( $\alpha \mathrm{UMa}$ ) and the prototype $\delta$ Cep. The accuracy for the former is $1.6 \%$ (van Leeuwen et al. 2007), and for the latter similar to the accuracy of the FGS HST parallax, namely $5.2 \%$ versus 4.1\% (Mérand et al. 2005).

The Baade-Wesselink method (BW, Baade 1926; Wesselink 1946) provides an independent empirical approach to measuring 
Table 1. Intrinsic parameters, mean NIR magnitudes, and mean radial velocities for the target Cepheids.

\begin{tabular}{lcccccccccc}
\hline \hline NAME & $\alpha(\mathrm{J} 2000)^{a}$ & $\delta(\mathrm{J} 2000)^{a}$ & $\log P$ & $E(B-V)^{b}$ & $\langle J\rangle^{c} \pm \sigma(J)$ & $\langle H\rangle^{c} \pm \sigma(H)$ & $\langle K\rangle^{c} \pm \sigma(K)$ & $N_{\mathrm{s}}{ }^{d}$ & $\left\langle v_{\mathrm{r}}\right\rangle^{e}$ & $\Delta v_{\mathrm{r}}{ }^{e}$ \\
\hline V340 Ara & 164519 & -512033 & 1.32 & 0.574 & $7.382 \pm 0.011$ & $6.809 \pm 0.007$ & $6.619 \pm 0.008$ & $25+2$ & $-80.8 \pm 1.2$ & 59.1 \\
UZ Sct & 183122 & -125500 & 1.17 & 1.071 & $7.502 \pm 0.049$ & $6.818 \pm 0.042$ & $6.564 \pm 0.045$ & $25+2$ & $40.2 \pm 0.5$ & 49.4 \\
AV Sgr & 180449 & -224300 & 1.19 & 1.267 & $6.909 \pm 0.040$ & $6.081 \pm 0.035$ & $5.758 \pm 0.033$ & $25+1$ & $19.3 \pm 1.8$ & 59.3 \\
VY Sgr & 181205 & -204200 & 1.13 & 1.283 & $7.174 \pm 0.069$ & $6.375 \pm 0.046$ & $6.068 \pm 0.040$ & $25+2$ & $16.0 \pm 1.8$ & 59.2 \\
\hline
\end{tabular}

Notes. ${ }^{(a)}$ Cepheid coordinates: units of right ascension are hours, minutes, and seconds; units of declination are degrees, arcminutes, and arcseconds. ${ }^{(b)}$ Reddening according to Fernie et al. (1995). ${ }^{(c)}$ Mean NIR magnitudes. ${ }^{(d)}$ Number of spectra collected with FEROS at the $2.2 \mathrm{~m}$ MPG/ESO telescope. ${ }^{(e)}$ Mean radial velocity and velocity amplitude $\left(\mathrm{km} \mathrm{s}^{-1}\right)$.

Cepheid absolute distances and can be applied to variable stars. This method relies on two observables: the radial velocity $\left(v_{\mathrm{r}}\right)$ and the variation in the angular diameter $(\theta)$. The latter parameter has been historically substituted by the variation in color along the pulsation cycle. However, direct measurements of the Cepheid angular diameter were provided by Kervella et al. (2004b) using the Very Large Telescope Interferometer (VLTI). In particular, the VLT INterferometer Commissioning Instrument (VINCI) gave the unique opportunity to provide angular diameter measurements along the pulsation cycle for seven Cepheids. These pioneering measurements encouraged a detailed comparison between theory and observations concerning limb darkening and the atmosphere of variable stars (Marengo et al. 2003; Nardetto et al. 2007), but the number of Cepheids for which these measurements are available remains very limited.

To overcome the difficulties in the interferometric measurement of the angular diameter, several variants of the BW method have been developed. Among them, the methods based on the surface-brightness (SB) relations search for correlations between variations in color and variations in angular diameters. This method can be applied to Cepheids for which accurate radial velocities and multiwavelength light curves are available. Storm et al. (2004), Kervella et al. (2004a), and Groenewegen (2007, hereafter G07) derived these relations using an opticalNIR $(V-K)$ color (IRSB), which enables the highest precision to be achieved in the derived quantities.

However, the most relevant limitation of typically adopted BW methods is the value of the projection factor ( $p$-factor). This parameter links radial velocity variations to radius changes but still lacks strong theoretical and empirical constraints (Nardetto et al. 2009). It can be empirically estimated using Cepheids for which accurate interferometric angular diameter measurements, radial velocity curves, and trigonometric parallaxes are available. This approach was applied to $\delta$ Cep by Mérand et al. (2005) using the new optical interferometric measurements obtained with the CHARA Array, the FGS HST trigonometric parallax by Benedict et al. (2007), and the radial velocities available in the literature. The $p$-factor that they found, $p=1.27 \pm 0.06$, agrees well with theoretical predictions by Nardetto et al. (2004). G07 found that the use of a constant $p$-factor $(p=1.27 \pm 0.05)$ for six Galactic Cepheids with interferometrically measured angular diameter variations and known distances, agrees quite well with HST parallaxes. He found that a strong period dependence of the $p$-factor $(p \sim-0.15 \cdot \log P$, Gieren et al. 2005), could also be ruled out. However, a moderate period dependence $(p \sim-0.03 \cdot \log P$ ), as suggested by Gieren et al. (1993, 1997, 1998), Barnes et al. (2003), Storm et al. (2004), and Nardetto et al. (2009) $(p \sim-0.08 \cdot \log P)$, is consistent with currently available data (G07).
We apply the BW method using the latest calibration of the IRSB relation (Groenewegen 2010, hereinafter G10, in preparation) to estimate the distance of four metal-rich Galactic Cepheids. In particular, we use new radial velocity measurements, new accurate NIR $(J, H, K)$ light curves, and $V$-band light curves available in the literature (Berdnikov 1992). We discuss in Sect. 4 the iron abundance of the four target Cepheids using Fe I and Fe II lines. Section 5 deals with the pulsation amplitude of metal-rich Cepheids, while in Sect. 6 we summarize current findings and outline future developments of this project.

\section{Photometric and spectroscopic data}

The selected stars are the most metal-rich Cepheids in the large sample (113 objects) collected by Andrievsky et al. (2002a,b,c, 2004). Positional and physical parameters for the selected Cepheids are listed in Table 1.

\subsection{Optical and NIR data}

To provide accurate estimates of their distances, we use the most recent calibration of the IRSB relation (Barnes et al. 1976; Laney \& Stobie 1995; Fouqué \& Gieren 1997; Storm et al. 2004; G10), and one of us (CDL) collected accurate multi-epoch, multi-band NIR data at SAAO for the four targets. The typical uncertainty in individual phase points ranges from 0.005 to 0.007 mag for $K<6 \mathrm{mag}$, diminishing to about 0.012 at $K=8.6 \mathrm{mag}$. Figure 1 shows the $J, H$, and $K$-band light curves for the target Cepheids. The intrinsic scatter $(\sigma)$ in the fit with a cubic spline (red line) and the luminosity amplitude are also labeled. The mean NIR $(J, H, K)$ magnitudes were estimated by obtaining the time average of the magnitudes and are listed in Table 1. The typical uncertainties are on the order of a few hundredths of a magnitude.

For three of the four targets (AV Sgr, UZ Sct, VY Sgr) we present detailed optical light curves collected in the Walraven bands (Walraven et al. 1964; Lub \& Pel 1975; Pel 1976; Lub \& Pel 1977). However, to derive the Baade-Wesselink solution we decided to use more recent $V$-band light curves available in the literature (Berdnikov 1992), since the accuracy improves if we consider photometric and radial velocity data that are close in time. The large sample of Cepheids with Walraven photometry will be used in Sect. 5, where we discuss the period-amplitude diagram.

\subsection{Spectroscopic data}

In addition to photometric data, we acquired for the target Cepheids accurate multi-epoch, high-resolution $(R \sim 30000)$ spectra with FEROS mounted on the $2.2 \mathrm{~m} \mathrm{MPG/ESO} \mathrm{telescope}$ 
S. Pedicelli et al.: New Baade-Wesselink distances and radii for 4 metal-rich Galactic Cepheids

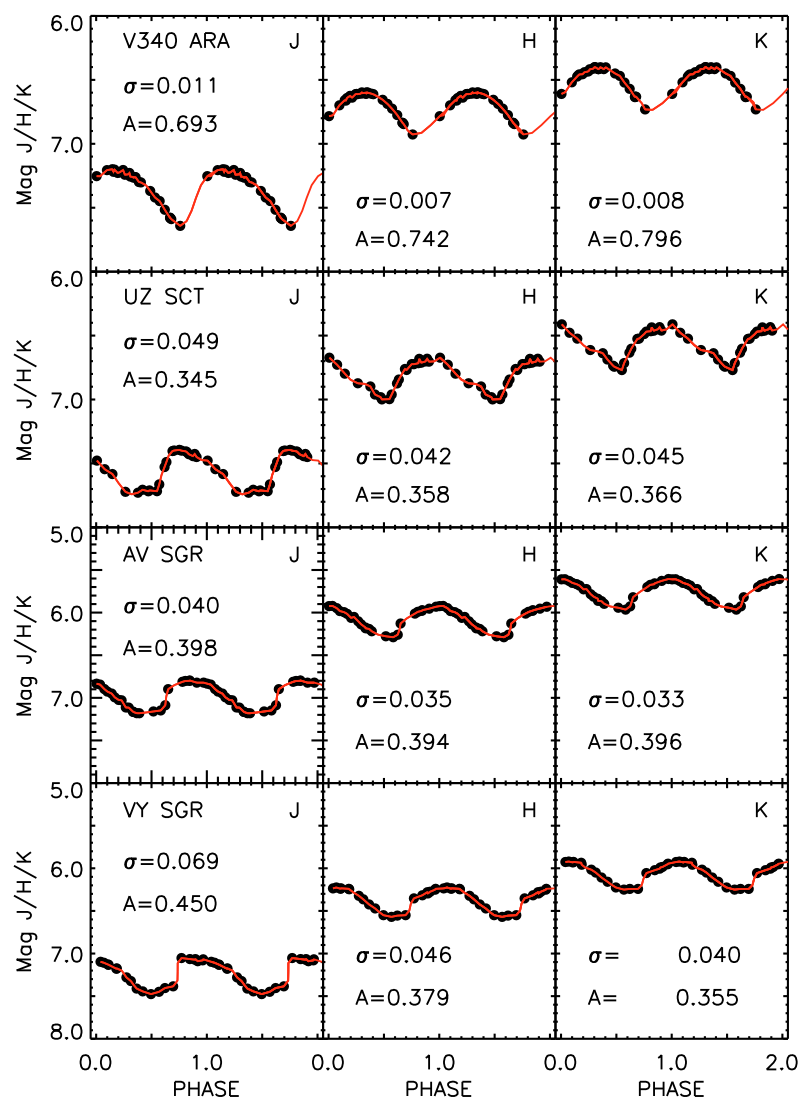

Fig. 1. From left to right $J, H, K$-band light curves for the four metalrich selected Cepheids. In each panel, we also plot the intrinsic scatter $(\sigma)$ of the fit with a cubic spline (red line) and the luminosity amplitude.

covering the entire pulsational cycle. The goal was to provide individual radial velocity measurements with an accuracy of $1 \mathrm{~km} \mathrm{~s}^{-1}$ for $\approx$ two dozen epochs. The number of epochs is a strong requirement to meet, using $\mathrm{BW}$ methods, the nominal accuracy of $5 \%$ in distance. The spectroscopic sample includes more than 25 spectra per Cepheid (see Col. 9 in Table 1). These spectra were collected between May and September 2005. The typical exposure time was $\sim 320 \mathrm{~s}$, and the quality of the individual spectra is quite good (signal-to-noise ratio hereafter $S / N$ of about 70) allowing us to reach the accuracy required in the radial velocity measurements. Radial velocity measurements were performed using fitline a semi-interactive routine developed by one of us (PF, François et al. 2007), which is based on a cross-correlation between the lines of each spectrum and a given linelist. The reader interested in a more detailed description of fitline is referred to Lemasle et al. $(2007,2008)$. We note that to deal properly with metal-rich Cepheids the iron linelist provided by Romaniello et al. (2008) was supplemented with three dozen weak Fe II lines (see Table 2). Figure 2 shows the individual radial velocity measurements and the fit with a cubic spline (red lines). The mean radial velocities $\left(\left\langle v_{\mathrm{r}}\right\rangle\right)$ and the velocity amplitudes $\left(\Delta v_{\mathrm{r}}\right)$ based on the spline fit are also labeled (see Cols. 10 and 11 of Table 1$)$.

The target Cepheids are among the most metal-rich Galactic Cepheids and, as expected, they are located close to the inner edge of the thin disk (Pedicelli et al. 2009). To provide accurate iron abundance measurements, we also collected for each target 1-2 high $S / N$ spectra with FEROS at the $2.2 \mathrm{~m} \mathrm{MPG/ESO}$ telescope (see red asterisks in Fig. 2 and Table 1). These spectra were collected by one of us (PF) at the end of March 2007.
Table 2. New weak Fe II line list added to the Fe I and Fe II line list of Romaniello et al. (2008).

\begin{tabular}{cccc}
\hline \hline$\lambda[\AA]$ & Ion & $E P$ & $\log g_{\mathrm{f}}$ \\
\hline 4893.82 & Fe II & 2.83 & -4.45 \\
4923.93 & Fe II & 2.88 & -1.35 \\
4993.35 & Fe II & 2.81 & -3.56 \\
5100.66 & Fe II & 2.81 & -4.16 \\
5132.67 & Fe II & 2.81 & -3.95 \\
5197.58 & Fe II & 3.23 & -2.23 \\
5234.63 & Fe II & 3.22 & -2.22 \\
5256.94 & Fe II & 2.89 & -4.25 \\
5325.56 & Fe II & 3.22 & -3.18 \\
5414.07 & Fe II & 3.22 & -3.54 \\
5425.26 & Fe II & 3.2 & -3.27 \\
5534.85 & Fe II & 3.24 & -2.75 \\
5932.06 & Fe II & 3.2 & -4.81 \\
7135.02 & Fe II & 6.21 & -2.60 \\
7301.56 & Fe II & 3.89 & -3.68 \\
7310.22 & Fe II & 3.89 & -3.36 \\
7672.37 & Fe II & 3.15 & -5.19 \\
7777.11 & Fe II & 3.20 & -5.24 \\
7801.24 & Fe II & 5.91 & -2.94 \\
7841.39 & Fe II & 3.90 & -3.72 \\
8250.34 & Fe II & 5.22 & -3.29 \\
8264.72 & Fe II & 6.81 & -2.08 \\
8324.96 & Fe II & 6.22 & -2.75 \\
8330.59 & Fe II & 6.22 & -2.35 \\
8490.08 & Fe II & 9.74 & 0.34 \\
8981.13 & Fe II & 6.72 & -2.18 \\
8990.39 & Fe II & 6.23 & -2.38 \\
9095.11 & Fe II & 9.65 & 0.27 \\
9122.92 & Fe II & 9.85 & 0.53 \\
9132.37 & Fe II & 9.85 & 0.51 \\
9187.16 & Fe II & 9.70 & 0.25 \\
9244.74 & Fe II & 6.22 & -2.40 \\
9297.27 & Fe II & 9.65 & 0.41 \\
9464.88 & Fe II & 6.09 & -2.54 \\
9572.60 & Fe II & 5.82 & -2.87 \\
9843.19 & Fe II & 6.14 & -2.60 \\
9849.74 & Fe II & 6.73 & -2.30 \\
9956.31 & Fe II & 5.48 & -2.95 \\
\hline & & &
\end{tabular}

The typical exposure time is $\sim 1800 \mathrm{~s}$ and their $S / N$ ratio ranges from 150 to 300 . The abundance analysis of the Cepheids with two high $S / N$ spectra was performed on the coadded spectrum, since they were collected close in time. Figure 3 shows the coadded spectra for the four selected Cepheids in the wavelength range between 5930 and $6000 \AA$. The vertical dashed and dotted lines indicate the Fe I $(\lambda=5930.17$, 5934.66, $5956.70,5976.78,5983.69,5984.79,5987.05 \AA)$ and Fe II $(\lambda=$ $5932.06,5991.37 \AA$ ) lines, respectively. The $S / N$ of either the individual (AV Sgr) or coadded spectra is also labeled.

\section{BW method}

The BW method adopted to estimate distances and radii was discussed in (Groenewegen 2004, 2007, 2008). We briefly mention the key points of the method and the differences from the original approach. According to the definition of quasi-monochromatic surface brightness, we can write $M_{V}-S_{V}+5 \times \log \left(R / R_{\odot}\right)=$ const. The absolute magnitude, and in turn the distance, can be found by differentiating this equation with respect to the pulsation phase, by multiplying the result for a color index $-(V-K)_{0}$ - and then by integrating over the entire pulsation cycle. The radial velocity is tightly correlated with the pulsation velocity and 


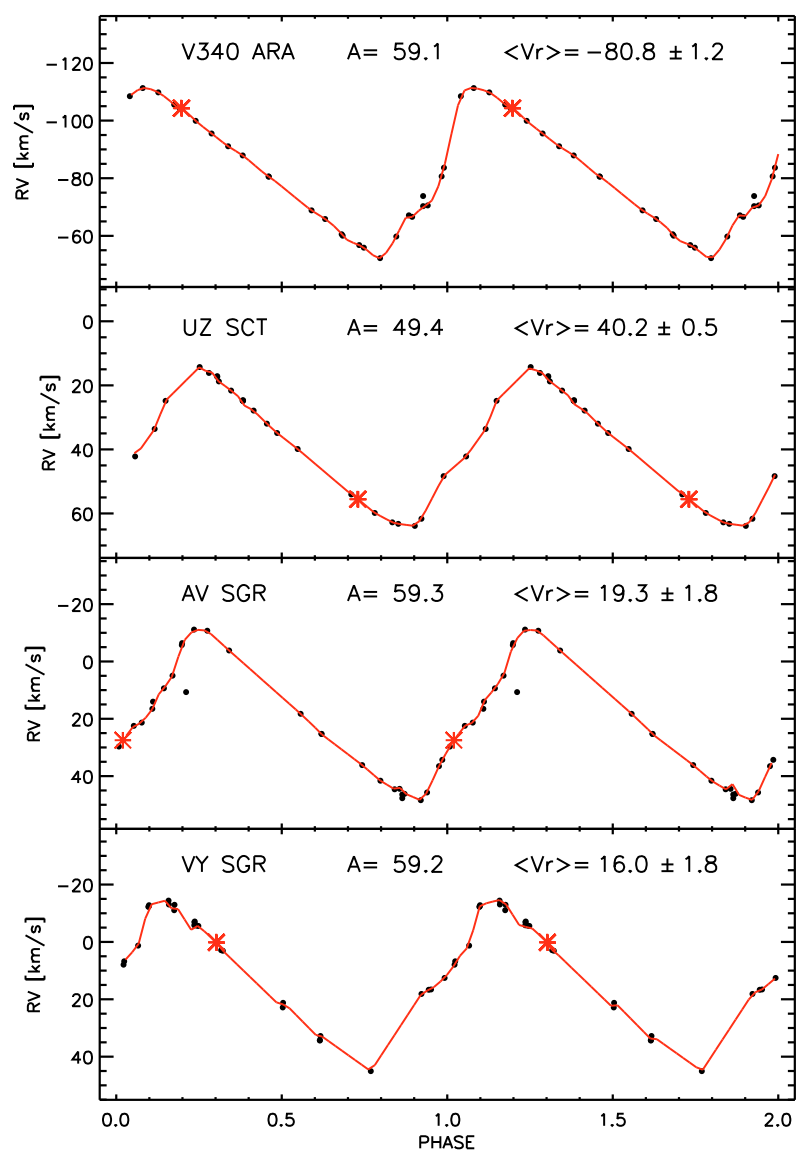

Fig. 2. Radial velocity curves for the four selected Cepheids, measured by cross-correlating a line list (see text). The red asterisks display the radial velocities based on the high $S / N$ spectra adopted for measuring the iron abundance. The amplitude and the mean radial velocity $\left(\mathrm{km} \mathrm{s}^{-1}\right)$ are labeled.

the curve can be integrated to obtain the radius variation as a function of time (phase)

$\Delta R(t, \delta \theta)=-p \int_{t_{0}}^{t+P \delta \theta}\left(v_{\mathrm{r}}(t)-v_{\gamma}\right) \mathrm{d} t$,

where $P$ is the pulsation period, $p$ the projection factor, $v_{\gamma}$ the systematic radial velocity, and $\delta \theta$ accounts for a phase shift between the radial velocity curve and the angular diameter changes measured either interferometrically or by means of the SB relation. Eventually, we end up with the equation $\Theta(t)=$ const. $\times$ $\left[R_{0}+\Delta R(t, \delta \theta)\right] / d$, where $\Theta(t)$ is the angular diameter in mas, $R_{0}$ the stellar radius in solar radii, and $d$ the distance in parsec. We measure the apparent radial velocity $v_{\mathrm{r}}$, i.e. the Doppler shift of absorption lines in the stellar atmosphere, projected along the line of sight and integrated over the stellar disk. To obtain the pulsational velocity, we use the $p$-factor (see Col. 2 in Table 3) derived from Nardetto et al. (2009)

$p=1.31 \pm 0.06-0.08 \pm 0.05 \cdot \log (P)$.

Integration of the pulsational velocity over the entire pulsation cycle provides an estimate of the linear radius variation. The angular diameter variation was derived using the IRSB method and the $(V-K)$ color. The calibration adopted in this investigation was derived by $\mathrm{G} 10$

$\log \theta_{0}=0.2692(V-K)_{0}+0.5298$.

The above relation and Eq. (1) give, once integrated over the pulsation cycle, individual Cepheid distances.

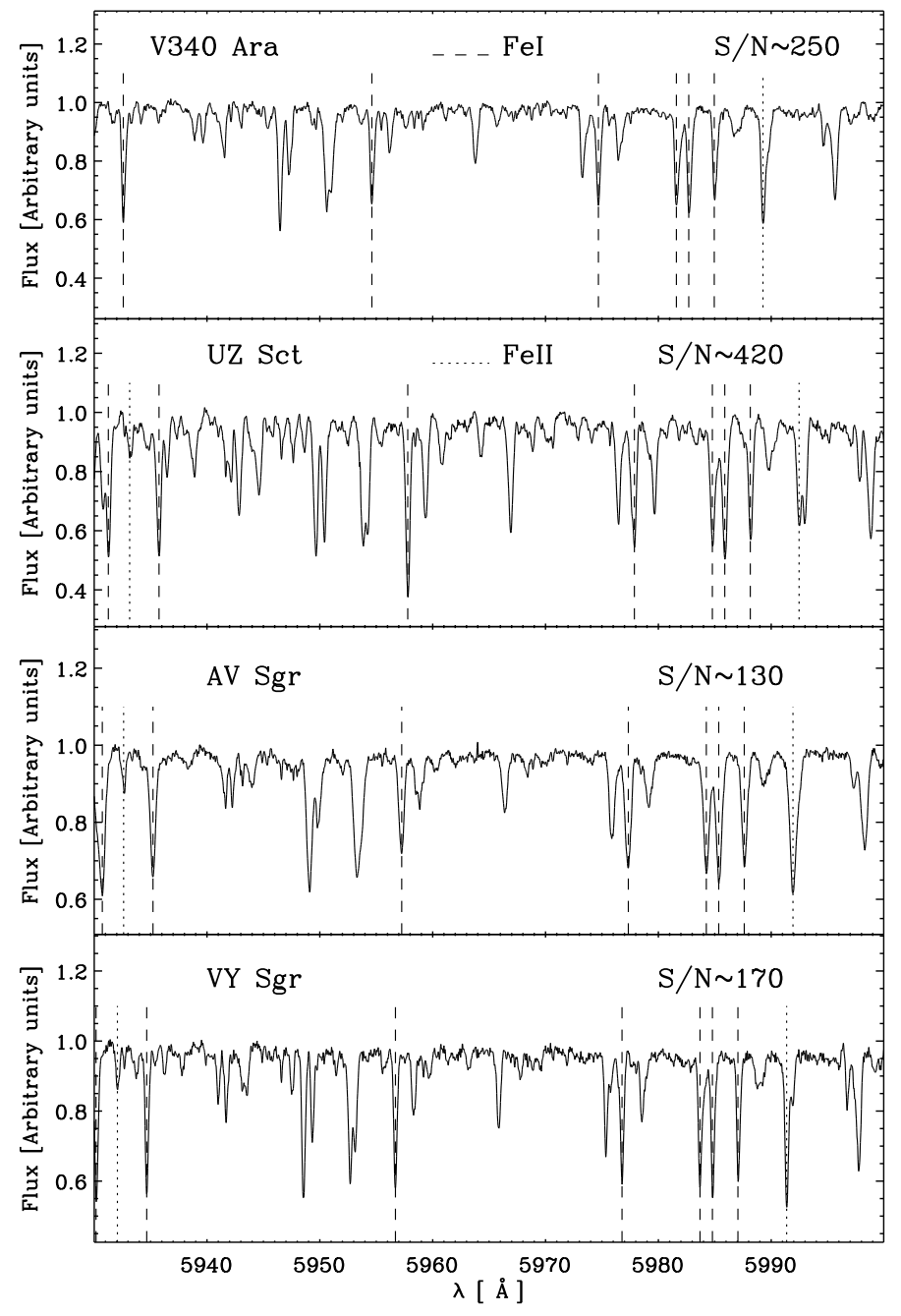

Fig. 3. High $S / N$ spectra for the four selected Cepheids in a limited wavelength range (5930-6000 ̊). Vertical dashed and dotted lines mark Fe I and Fe II lines. In each panel is also plotted the $S / N$ the of either individual (AV Sgr) or coadded spectra.

\subsection{Cepheid distances}

Figure 4 shows from left to right the application of the BW method to V340 Ara, UZ Sct, AV Sgr, and VY Sgr. For each Cepheid, we plot the variation in the angular diameter with phase (bottom panels) and the change in angular diameter derived from the IRSB relation (see Eq. (3)) versus the change in radius (top panels) obtained by integration of the $R V$ curve (see Eq. (1)). The absolute magnitudes were inferred in a self-consistent way from the Fourier fit to the data. We note that the fit in the phase interval $0.8-1.0$ is often poor. This limitation of the BW method was known before (Storm et al. 2004). It was argued that non-LTE effects and an increase in the micro turbulence during these phases may change the atmospheric structure, and in turn hamper the use of a simple surface-brightness relation (Bersier et al. 1997). Table 3 lists the estimated distances and radii and the comparison with previous estimates. The errors listed in Cols. 3 and 4 of Table 3 give the uncertainties (one $\sigma$ ) on distance and radius for the target Cepheids. They account not only for the errors in the fit, but also for the errors estimated running several Monte Carlo simulations by artificially changing individual errors in $V$, $K$, and $R V$ measurements. To compare the above distances with similar estimates available in the literature we adopted the $J$, 
Table 3. Distances and radii according to the BW-analysis of the four selected Cepheids.

\begin{tabular}{lccrrccc}
\hline \hline Name & $p$ & $d(\mathrm{pc})$ & $R / R_{\odot}$ & $d(\mathrm{pc})_{\mathrm{PL}}{ }^{a}$ & $d(\mathrm{pc})_{\mathrm{PL}}{ }^{b}$ & $\left(R / R_{\odot}\right)_{\mathrm{G} 07}{ }^{c}$ & $\left(R / R_{\odot}\right)_{\text {theo }}{ }^{d}$ \\
\hline V340 Ara & 1.205 & $3890 \pm 126 \pm 547$ & $100.2 \pm 3.2 \pm 17.3$ & $3154 \pm 686$ & $3754 \pm 1156$ & $109.5 \pm 1.1$ & $115 \pm 1$ \\
UZ Sct & 1.217 & $3176 \pm 116 \pm 152$ & $82.1 \pm 3.0 \pm 3.5$ & $3117 \pm 871$ & $3540 \pm 1177$ & $86.4 \pm 1.1$ & $91 \pm 1$ \\
AV Sgr & 1.215 & $2302 \pm 72 \pm 123$ & $93.4 \pm 2.9 \pm 4.2$ & $3036 \pm 792$ & $3326 \pm 1063$ & $89.2 \pm 1.1$ & $94 \pm 1$ \\
VY Sgr & 1.219 & $2546 \pm 53 \pm 134$ & $86.5 \pm 1.8 \pm 3.9$ & $2300 \pm 663$ & $2533 \pm 849$ & $81.1 \pm 1.1$ & $86 \pm 1$ \\
\hline
\end{tabular}

Notes. ${ }^{(a)}$ Mean $J H K$-distances based on empirical NIR PL relations (Fouqué et al. 2007). ${ }^{(b)}$ Mean $J H K$-distances based on theoretical NIR PL relations (Caputo, private communication). ${ }^{(c)}$ Radius estimate according to the empirical PR relation by G07. ${ }^{(d)}$ Radius estimate according to the theoretical PR relation by Petroni et al. (2003).
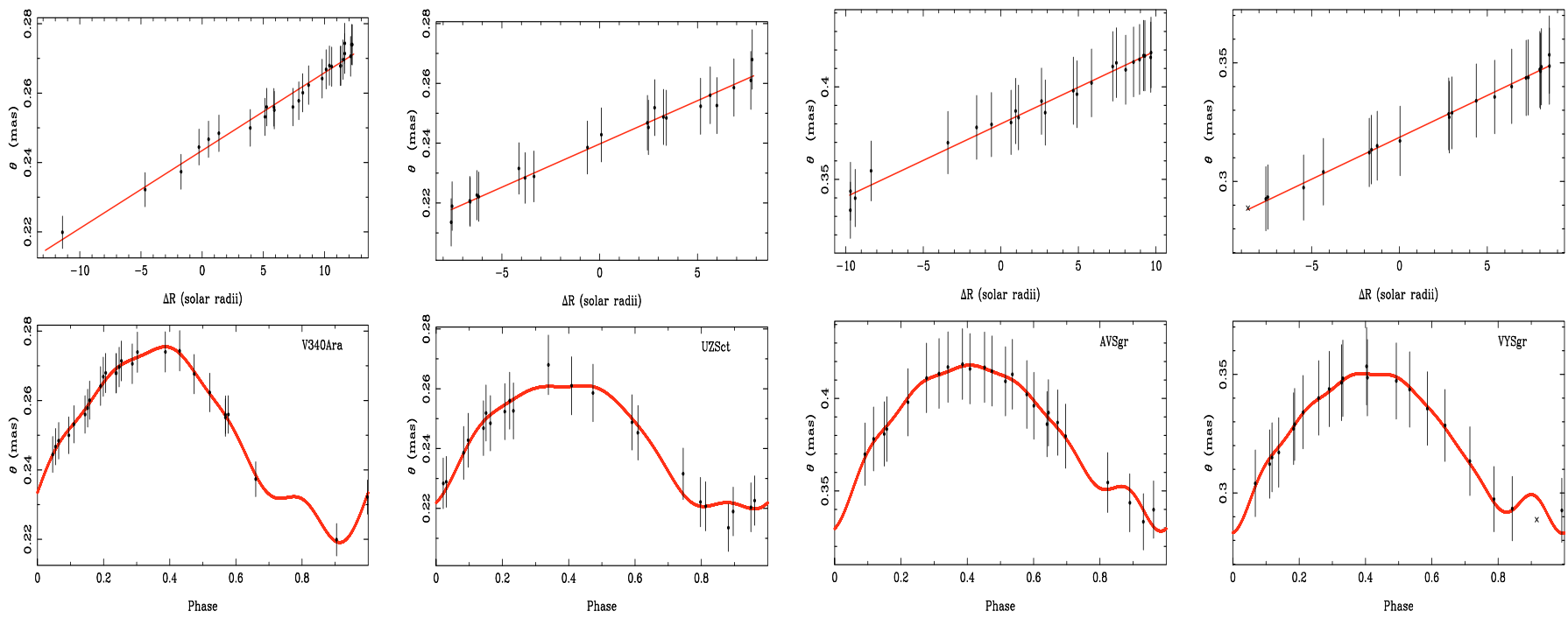

Fig. 4. From left to right: V340 Ara, UZ Sct, AV Sgr, and VY Sgr. For each star, the top panels show the linear-bisector fit to the angular diameter as a function of radial displacement. The bottom panels show the angular diameter against phase. Crosses indicate data-points not included in the fit.

$H$ and $K$-band PL relations for Galactic Cepheids recently provided by Fouqué et al. (2007). The mean of the three individual distances together with their errors for the target Cepheids are listed in Cols. 5 of Table 3. The errors account for the uncertainty in both the mean magnitudes and the PL relations. The comparison indicates that distances based on both the BW method and NIR PL relation agree to within $1 \sigma$. The uncertainties in the $\mathrm{BW}$ distances have been typically determined by taking into account the errors in both the fit and those estimated using Monte Carlo simulations to have values from $6 \%$ (UZ Sct, AV Sgr, VY Sgr) to 14\% (V340 Ara), while the uncertainties in the distances based on the empirical NIR PL relations range from $22 \%$ to $34 \%$.

The NIR PL relations provided by Fouqué et al. (2007) do not account for a possible dependence of the NIR PL relation on the metal content. We consider Cepheids characterized by super-solar iron abundances. Therefore, we estimated the Cepheid distances using theoretical NIR PL relations including both metal-intermediate and metal-rich Cepheid models (F. Caputo, private communication). Data listed in Col. 6 of Table 3 indicate that distances based on these theoretical NIR PL relations also agree to within $1 \sigma$ with the $\mathrm{BW}$ estimates. We note that the distances and their uncertainties based on theoretical PL relations are larger than the empirical ones, since the former relations were derived including all the fundamental models with abundances ranging from $Z=0.001$ to $Z=0.04$ and different helium contents (Bono et al. 2010, in prep.).

The mean Cepheid radii listed in Col. 4 of Table 3 agree quite well with the mean radii inferred from empirical period-radius (PR) relation for Galactic Cepheids recently provided by G07 (see Col. 7 in Table 3). We note that this PR relation is based on data for six Galactic Cepheids with known distances (Benedict et al. 2007) and interferometrically measured angular diameter variations (Kervella et al. 2004b; Mérand et al. 2005). A similar result was found when comparing with radii based on the predicted PR relation for Galactic Cepheids provided by Petroni et al. (2003), assuming a solar chemical composition. Current radii agree on average to within $10 \%$ (two $\sigma$ ) with the predicted and empirical PR relations.

\section{Cepheid iron abundances}

To measure the iron abundances of the target Cepheids, we followed the same approach suggested by Lemasle et al. (2007), which relies on three different types of input data:

- Line list: We adopted the line list of Romaniello et al. (2008), which includes $275 \mathrm{Fe}$ I lines and $37 \mathrm{Fe}$ II lines covering the FEROS spectral range. However, the target Cepheids are metal-rich and a good fraction of the Fe II lines are saturated. To provide robust estimates of intrinsic parameters and hence accurate measurements of iron abundances, we supplemented the line list of Romaniello et al. (2008) with 39 new weak Fe II lines (see Table 2).

- Equivalent width: The measurement of the equivalent widths (EW) of the iron lines was performed using fitline. This code uses a Gaussian fit, which is defined by the four parameters of central wavelength, width, depth, and continuum value of the individual lines. The initial value of the Gaussian 
Table 4. Intrinsic parameters and iron abundances for the target Cepheids.

\begin{tabular}{llccccccc}
\hline \hline Name & $T_{\text {eff }}(\mathrm{LDR})$ & $\log g$ & $v_{\mathrm{t}}$ & $T_{\text {eff }}(\mathrm{Model})$ & {$[\mathrm{Fe} \mathrm{I} / \mathrm{H}]$} & {$[\mathrm{Fe} \mathrm{II} / \mathrm{H}]$} & $\langle[\mathrm{Fe} / \mathrm{H}]\rangle$ & {$[\mathrm{Fe} / \mathrm{H}]_{\text {And }}$} \\
\hline V340 Ara & $5425 \pm 146$ & 0.2 & 3.5 & 5475 & $0.38 \pm 0.17$ & $0.42 \pm 0.26$ & $+0.40 \pm 0.21$ & $+0.31 \pm 0.10$ \\
UZ Sct & $4790 \pm 95$ & 0.6 & 3.7 & 4850 & $0.35 \pm 0.17$ & $0.35 \pm 0.27$ & $+0.35 \pm 0.21$ & $+0.33 \pm 0.10$ \\
AV Sgr & $5407 \pm 53$ & 0.9 & 4.2 & 5450 & $0.26 \pm 0.12$ & $0.28 \pm 0.12$ & $+0.27 \pm 0.12$ & $+0.34 \pm 0.10$ \\
VY Sgr & $5268 \pm 146$ & 1.0 & 3.2 & 5400 & $0.38 \pm 0.17$ & $0.36 \pm 0.26$ & $+0.35 \pm 0.21$ & $+0.26 \pm 0.10$ \\
\hline
\end{tabular}

parameters is fixed by randomly selecting the four parameters. The "genetic" algorithm then computes the $\chi^{2}$ between the observed line and the expected Gaussian profile and the new set of Gaussian parameters among the 20 best fit solutions of the previous "generation" by applying random modifications to the values of the parameters ("mutation"). After 100-200 "generations", the algorithm provides the best-fit Gaussian parameters (lowest $\chi^{2}$ ) for each observed line. For the measurement of the iron abundance, we selected only lines with equivalent widths between 10 and $200 \mathrm{~m} \AA$. The lower limit was chosen to be a safe compromise between the spectral characteristics and the need for weak lines for an optimal abundance determination. The upper limit was fixed to avoid the saturated portion of the curve of growth.

- Stellar parameters: The determination of an accurate effective temperature is a critical point in the abundance determination. This requirement becomes even more important when we study variable stars, since the temperature estimate has to refer to the pulsation phase at which the spectrum was collected. In this analysis, the effective temperature $\left(T_{\text {eff }}\right)$ was estimated spectroscopically using the line depth ratios (LDR) method described in Kovtyukh \& Gorlova (2000). This technique has the advantage of being independent of interstellar reddening and minimally dependent on metallicity. These uncertainties plague other methods such as the integrated flux method or the color-temperature relations (Gray 1994; Krockenberger et al. 1998). This approach becomes even more relevant for the target Cepheids, since they are located close to the edge of the inner Galactic disk, and therefore they are characterized by high reddening values (see Col. 5 in Table 1). The estimated effective temperatures and their errors are listed in Col. 2 of Table 4. The surface gravity $(\log g)$ and the microturbulent velocity $\left(v_{\mathrm{t}}\right)$ were constrained by minimizing the $\log ([\mathrm{Fe} / \mathrm{H}])$ versus $\mathrm{EW}$ slope (using the Fe I abundance) and by imposing the ionization balance between Fe I and Fe II (see Cols. 3 and 4 in Table 4). These two procedures are tightly connected and require an iterative process. The initial values of the microturbulent velocity and surface gravity were fixed using typical Cepheid values $\left(v_{\mathrm{t}}=3 \mathrm{~km} \mathrm{~s}^{-1}, \log g=2\right.$, Andrievsky et al. $2002 b)$. For the ionization balance, we assume that it was fulfilled when the difference between $[\mathrm{Fe} \mathrm{I} / \mathrm{H}]$ and $[\mathrm{Fe} \mathrm{II} / \mathrm{H}]$ was smaller than the standard deviation in $[\mathrm{Fe} \mathrm{II} / \mathrm{H}]$ (typically, $\left.\sigma_{[\mathrm{Fe} / \mathrm{H}]} \sim 0.08-0.1 \mathrm{dex}\right)$. If this condition was satisfied by more than one value of $\log g$, we verified which value also satisfies the ionization balance within the standard deviation on $[\mathrm{Fe} \mathrm{I} / \mathrm{H}]$ (typically, $\sigma_{[\mathrm{Fe} / \mathrm{H}]} \sim 0.02 \mathrm{dex}$ ). The effective temperatures listed in Col. $5\left(T_{\text {eff }}(\right.$ Model $\left.)\right)$ are the final best-fit values of the atmosphere models adopted in the iterative process. To determine the errors in the microturbulent velocity and the surface gravity, we completed several iterations for each star, for each slightly modifying the values of these two intrinsic parameters that fulfill the requirements mentioned above. We have estimated that the intrinsic error in the microturbulent velocity is of the order of $0.1 \mathrm{~km} \mathrm{~s}^{-1}$, while the intrinsic error in the surface gravity is of the order of 0.10 dex.

Our final Fe I and Fe II abundances, and adopted stellar parameters, are listed in Table 4. Data listed in Col. 8 of this table indicate that the mean iron contents have weighted intrinsic uncertainties of the order of $0.20 \mathrm{dex}$, due to errors in the EW measurements, in the intrinsic parameters and in the number of unsaturated iron lines. This finding illustrates the difficulty in measuring the iron abundance of metal-rich Cepheids. The intrinsic accuracy can certainly be improved using high a $S / N$, multi-epoch spectra of the same targets, since empirical evidence suggests that Cepheid elemental abundances depend minimally on the pulsation phase (Luck \& Andrievsky 2004; Kovtyukh et al. 2005). Finally, we note that current iron abundances agree, within one $\sigma$, with the abundances provided by Andrievsky et al. (2002b) using a similar approach (see last column in Table 4).

\section{Period-amplitude diagram}

The metal-rich regime of classical Cepheids was only marginally investigated, since until a few years ago iron measurements for these objects were not available. As we mentioned in Sect. 2, Walraven $V B L U W$ photometry is available for more than 160 Galactic Cepheids. The pulsation properties of these objects will be discussed in a forthcoming paper (Pedicelli et al. 2010). Interestingly, three out of the four target Cepheids belong to this sample, namely AV Sgr, UZ Sct, and VY Sgr. For these Cepheids, secured data for at least 30 phase points in five$V B L U W$ bands that properly cover the entire pulsation cycle. The intrinsic accuracy of individual measurements is on the order of a few millimag. The uncertainty in the mean magnitudes, estimated using a fit with a cubic spline, is at most of the order of a few hundredths of a magnitude. To investigate the possible correlation between luminosity amplitude and iron abundance, the Walraven $B_{\mathrm{W}}, V_{\mathrm{W}}$-band photometric data were transformed into the Johnson $B_{\mathrm{J}}, V_{\mathrm{J}}$-band data using the relations

$V_{\mathrm{J}}=6.886^{\mathrm{mag}}-2.5 V_{\mathrm{W}}-0.1916(V-B)_{\mathrm{W}}$

$$
\begin{array}{r}
(B-V)_{\mathrm{J}}=2.7947(V-B)_{\mathrm{W}}-1.2052(V-B)_{\mathrm{W}}^{2} \\
+0.6422(V-B)_{\mathrm{W}}^{3}-0.0100 .
\end{array}
$$

Accurate iron abundance measurements for Walraven Cepheids are available for 77 objects, while for 67 objects metallicity estimates are available based on the Walraven metallicity index (Pedicelli et al. 2008, and references therein). This sample was supplemented with 115 Cepheids for which accurate iron abundances exist in the literature, based on high resolution spectra (Andrievsky et al. 2002a,b,c, 2004; Lemasle et al. 2007; Sziládi et al. 2007; Lemasle et al. 2008; Romaniello et al. 2008). We ended up with a sample of 259 Galactic Cepheids and the top panel of Fig. 5 shows the metallicity distribution. To provide accurate estimates of the mean metallicity and their spread in 


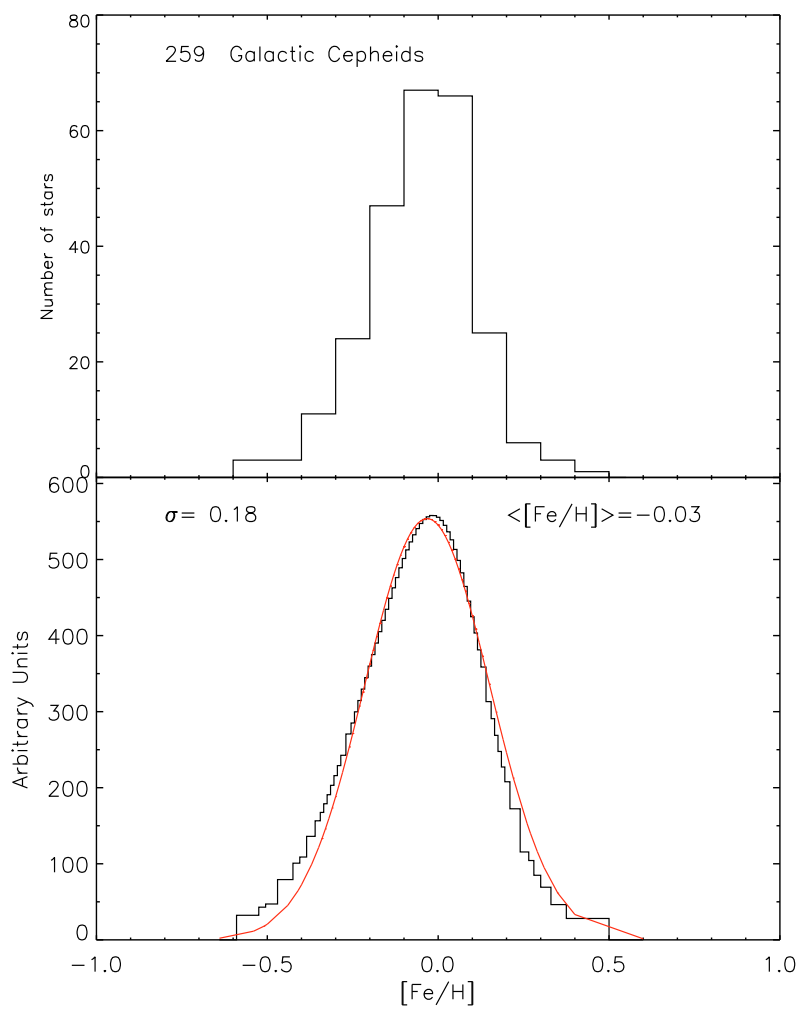

Fig. 5. Top - Metallicity distribution of Galactic Cepheids. Bottom Same as the top, but the metallicity distribution was smoothed using a Gaussian kernel with standard deviation equal to the metallicity uncertainty of individual Cepheids. The red line shows the Gaussian fit of the metallicity distribution. The mean and the $\sigma$ are also labeled.

metallicity of the Galactic disk, we created a Gaussian kernel with a $\sigma$ equal to the metallicity uncertainty of individual Cepheids. The metallicity distribution we obtained is plotted in the bottom panel of Fig. 5 and the red line shows the Gaussian fit. We found a solar mean metallicity $(\langle[\mathrm{Fe} / \mathrm{H}]\rangle \sim-0.03)$ and a sigma of 0.18 dex. These values agree quite well with similar metallicity distributions available in the literature (Chiappini et al. 2001; Cescutti et al. 2007). Our metallicity distribution is also asymmetric, and indeed the metal-poor tail is shallower than the metal-rich one. This finding agrees with predictions based on Galactic chemical evolution models (Holmberg et al. 2007; Yin et al. 2009).

To constrain the dependence of the pulsation properties on the metal content, we adopted the Bailey diagram, i.e. luminosity amplitude vs pulsation period. Data plotted in Figs. 6 and 7 show the $V, B$ luminosity amplitudes of the Cepheid sample adopted by Pedicelli et al. (2009). The amplitudes are based on either Walraven photometry - transformed into the Johnson system (see Sect. 2.1) - or data in the Galactic Cepheid catalog provided by Fernie et al. (1995). To constrain the possible dependence of the pulsation amplitude on the metal content, we selected three different sub-samples that are representative of the metal-poor $([\mathrm{Fe} / \mathrm{H}] \leq-0.30 \mathrm{dex})$ tail, the metal-rich $([\mathrm{Fe} / \mathrm{H}] \geq 0.13 \mathrm{dex})$ tail, and the peak metallicity $(-0.04 \leq[\mathrm{Fe} / \mathrm{H}] \leq-0.02 \mathrm{dex})$. The three samples roughly include two dozen Cepheids. Data plotted in the top panels of Figs. 6 and 7 indicate that both $V$ and $B$-band amplitudes are not correlated with the metal content. This feature does not agree with a well established theoretical and empirical evidence of RR Lyrae stars (Bono et al. 2007), i.e. the prototype of low-mass, helium burning radial variables located inside the so-called Cepheid instability strip. This

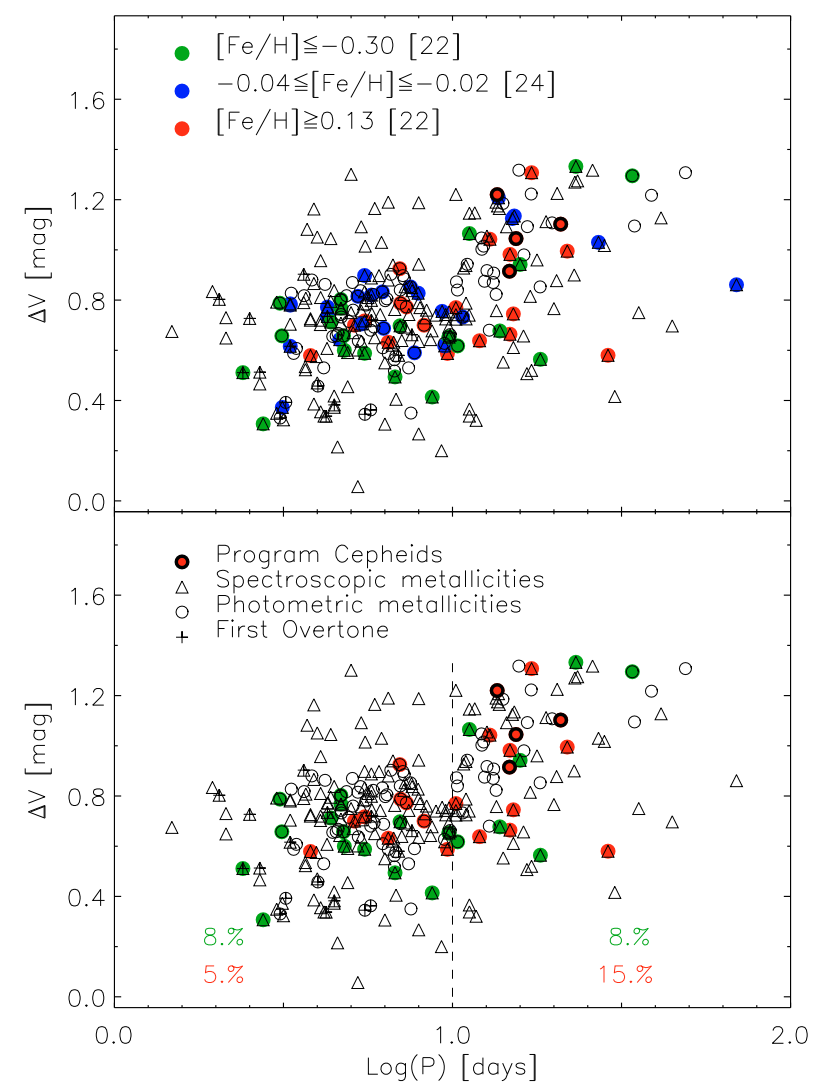

Fig. 6. Top $-V$-band amplitude versus period for a sample of 259 Galactic Cepheids. Triangles and circles display spectroscopic and photometric metallicities, respectively. Red and green dots mark metal-rich $([\mathrm{Fe} / \mathrm{H}] \geq 0.13)$ and metal-poor $([\mathrm{Fe} / \mathrm{H}] \leq-0.30)$ Cepheids, while the blue ones show Cepheids with iron abundances ranging from -0.04 to -0.02 dex. The selected metal-rich Cepheids are marked with a cross, while the pluses mark first overtone pulsators. Bottom - same as the top, but the vertical dashed line splits short- $(\log (P) \leq 1.0)$ and long-period $(\log (P)>1.0)$ Cepheids. The fractions of metal-poor and metal-rich Cepheids are also labeled.

difference can be partially explained with the empirical circumstance that Galactic RR Lyrae variables cover almost three dex in metal content, when moving from the halo to the bulge, while Galactic Cepheids in the disk cover only one dex. The Hertzsprung progression causes a systematic decrease in the pulsation amplitudes for periods across ten days (Payne-Gaposchkin 1951, 1954). The findings of both empirical (Andreasen \& Petersen 1987; Welch et al. 1997; Beaulieu 1998; Moskalik et al. 2000) and theoretical (Bono et al. 2000b) studies indicate that a decrease in metal content causes a systematic drift in the center of the Hertzsprung progression toward shorter periods. This implies that there is a reshuffle in the pattern of the luminosity amplitudes as a function of the pulsation period when studying Cepheids with different metal abundances. However, a spread in amplitude is also found at periods shorter and longer than the Hertzsprung progression. This indicates that the lack of a well defined correlation with metallicity might be caused by the dependence of the topology of the instability strip on the chemical composition (Pel \& Lub 1978; Bono et al. 1999, 2000a), by evolutionary effects, and by binarity (see Pedicelli et al. 2010, in preparation).

As a final test concerning the metallicity distribution of Galactic Cepheids, we divide the sample into short $(\log P \leq$ $1.0)$ and $\operatorname{long}(\log P>1.0)$ period objects. Data plotted in the bottom panels of Figs. 6 and 7 show that metal-rich 


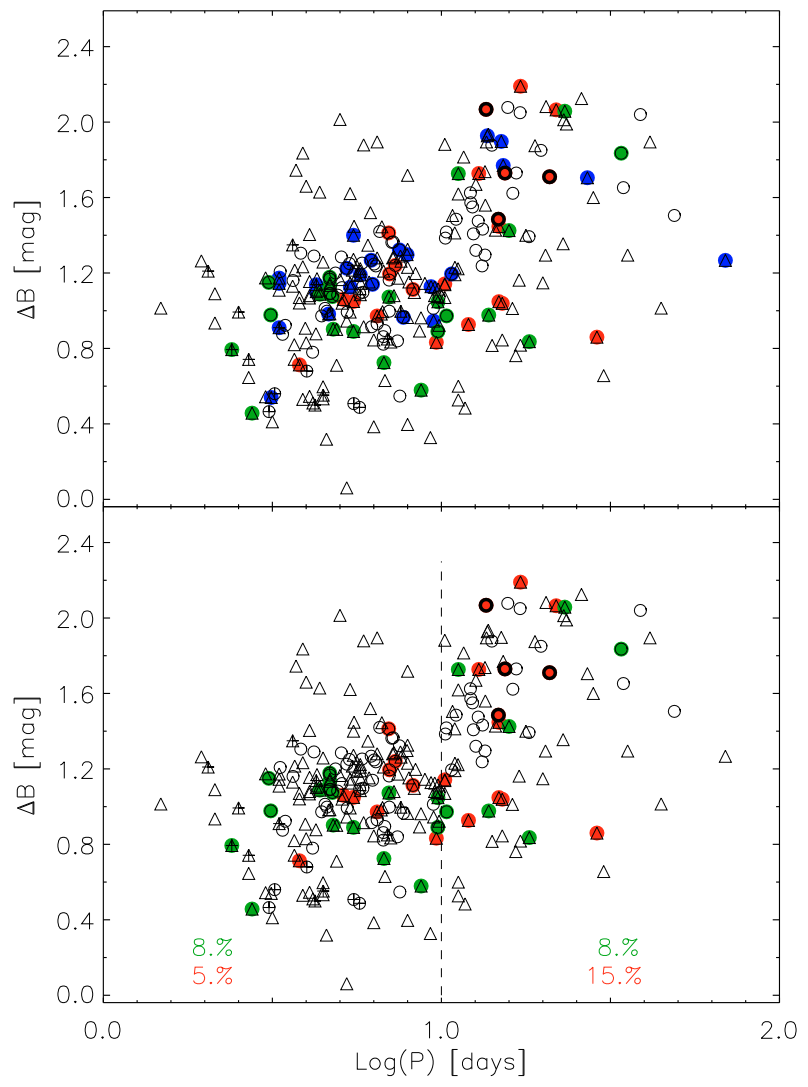

Fig. 7. Same as Fig. 6, but for the $B$-band amplitudes.

$([\mathrm{Fe} / \mathrm{H}] \geq-0.13$ dex $)$ Cepheids are more frequent among longthan among short-period Cepheids (15\% vs. $5 \%$ ) Cepheids. On the other hand, the metal-poor $([\mathrm{Fe} / \mathrm{H}] \leq-0.30$ dex $)$ Cepheids are equally distributed between the two groups. The evidence that the period distribution of classical Cepheids depends on the metal-content dates back to Gascoigne (1974, and references therein), who realized that the peak shifts toward shorter periods when moving from Galactic to Small Magellanic Cloud Cepheids. Subsequent evolutionary tracks for intermediate-mass stars showed that more metal-poor structures are characterized, at fixed mass, by blue loops that cover a larger temperature range. This means that the minimum Cepheid mass crossing the instability strip is lower for metal-poor than metal-rich systems. In consequence, a systematic drift in the period distribution of metal-rich Cepheids is expected. However, the period cut that we adopted is well beyond the short period cutoff of Galactic Cepheids, thus suggesting that the paucity of metal-rich Cepheids among short-period Cepheids is probably caused by an observational bias.

\section{Summary and conclusions}

We have determined accurate BW distances and radii for four metal-rich Cepheids, namely V340 Ara, UZ Sct, AV Sgr, and VY Sgr. Taken at face value, current distance estimates agree quite well with similar estimates based either on empirical (Fouqué et al. 2007) or theoretical NIR PL relations. However, the uncertainties affecting the BW distances, by summing in quadrature the errors in the fit and the errors estimated with the Monte Carlo simulations, are on average a factor of 3-4 smaller than for distances based on predicted and empirical NIR PL relation. The same outcome applies to the mean Cepheid radii, but the uncertainties in the BW radii, evaluated by summing in quadrature the errors in both the fit and Monte Carlo simulations, are on average a factor of two larger than for radii based on either the empirical or the theoretical PR relation provided by G07 and Petroni et al. (2003), respectively.

We also collected high-resolution, high $S / N$ ratio spectra to measure the iron abundances of the target Cepheids. Special care was taken to provide accurate estimates of intrinsic parameters (effective temperature, surface gravity, microturbulent velocity) directly from observed spectra. We performed detailed measurements of iron abundances using large samples of Fe I and Fe II lines. Current abundances indicate that selected Cepheids are super metal-rich and agree, within $1 \sigma$, with iron abundances provided by Andrievsky et al. (2002b) using a similar approach.

We adopted a sample of 259 Galactic Cepheids for which either spectroscopic iron measurements or metallicity estimates based on the Walraven metallicity index (Pedicelli et al. 2008) are available. In contrast to low-mass helium burning RR Lyrae stars, we found that classical Cepheids do not appear to exhibit in the Bailey diagram (luminosity amplitude versus pulsation period) a clear correlation between luminosity amplitude and metallicity. The lack of this correlation might be caused by the Hertzsprung progression. We also found that a good fraction of metal-rich $([\mathrm{Fe} / \mathrm{H}] \geq 0.13$ dex $)$ Cepheids are located among long-period $(\log P \geq 1.0)$ variables. However, for the moment this can only be considered as circumstantial evidence, since the current sample is probably affected by selection bias. Metal-rich Cepheids are located in the inner disk and are typically affected by high extinctions. The selected Cepheids have Galactocentric distances smaller than $6.5 \mathrm{kpc}$ and their reddening ranges from 0.6 to $1.3 \mathrm{mag}$. A detailed analysis of the pulsation properties of metal-rich Cepheids should be performed for more complete samples. However, classical Cepheids are excellent tracers of young stellar populations, and their pulsation properties and radial distribution across both the inner Galactic disk and the bar can provide robust constraints on bar-driven formation scenarios (van Loon et al. 2003; Debattista et al. 2004; Zoccali et al. 2006) on short (10-100 Myr) timescales.

Acknowledgements. We acknowledge an anonymous referee for his/her positive opinion concerning the content of this investigation. Two of us (S.P., G.B.) acknowledge ESO support (DGDF funds) for a stay in Garching, during which a good part of this paper was written. It is a pleasure to thank M. Zoccali for interesting discussions concerning the interaction between the Galactic disk and the bulge. We also thank G. Iannicola and I. Ferraro for their suggestions concerning Gaussian smoothing.

\section{References}

Andreasen, G. K., \& Petersen, J. O. 1987, A\&A, 180, 129

Andrievsky, S. M., Bersier, D., Kovtyukh, V. V., et al. 2002a, A\&A, 384, 140 Andrievsky, S. M., Kovtyukh, V. V., Luck, R. E., et al. 2002b, A\&A, 381, 32 Andrievsky, S. M., Kovtyukh, V. V., Luck, R. E., et al. 2002c, A\&A, 392, 491 Andrievsky, S. M., Luck, R. E., Martin, P., \& Lépine, J. R. D. 2004, A\&A, 413, 159

Baade, W. 1926, Astron. Nachr., 228, 359

Barnes, T. G., Evans, D. S., \& Parsons, S. B. 1976, MNRAS, 174, 503

Barnes, III, T. G., Jefferys, W. H., Berger, J. O., et al. 2003, ApJ, 592, 539 Beaulieu, J. P. 1998, Mem. Soc. Astron. Ital., 69, 21

Benedict, G. F., McArthur, B. E., Feast, M. W., et al. 2007, AJ, 133, 1810 Berdnikov, L. N. 1992, Astron. Astrophys. Trans., 2, 107 Bersier, D., Burki, G., \& Kurucz, R. L. 1997, A\&A, 320, 228

Bono, G., Marconi, M., \& Stellingwerf, R. F. 1999, ApJS, 122, 167 Bono, G., Castellani, V., \& Marconi, M. 2000a, ApJ, 529, 293 Bono, G., Marconi, M., \& Stellingwerf, R. F. 2000b, A\&A, 360, 245 Bono, G., Caputo, F., \& Di Criscienzo, M. 2007, A\&A, 476, 779 Bono, G., Caputo, F., Fiorentino, G., Marconi, M., \& Musella, I. 2008, ApJ, 684, 102

Cescutti, G., Matteucci, F., François, P., \& Chiappini, C. 2007, A\&A, 462, 943 
Chiappini, C., Matteucci, F., \& Romano, D. 2001, ApJ, 554, 1044

Debattista, V. P., Carollo, C. M., Mayer, L., \& Moore, B. 2004, ApJ, 604, L93

di Benedetto, G. P. 2008, MNRAS, 390, 1762

Feast, M. 1999, PASP, 111, 775

Fernie, J. D., Evans, N. R., Beattie, B., \& Seager, S. 1995, Inf. Bull. Variable Stars, 4148, 1

Fouqué, P., \& Gieren, W. P. 1997, A\&A, 320, 799

Fouqué, P., Arriagada, P., Storm, J., et al. 2007, A\&A, 476, 73

François, P., Depagne, E., Hill, V., et al. 2007, A\&A, 476, 935

Freedman, W. L., Madore, B. F., Gibson, B. K., et al. 2001, ApJ, 553, 47

Gascoigne, S. C. B. 1974, MNRAS, 166, 25P

Gieren, W. P., Barnes, III, T. G., \& Moffett, T. J. 1993, ApJ, 418, 135

Gieren, W. P., Fouqué, P., \& Gomez, M. I. 1997, ApJ, 488, 74

Gieren, W. P., Fouqué, P., \& Gomez, M. 1998, ApJ, 496, 17

Gieren, W., Storm, J., Barnes, III, T. G., et al. 2005, ApJ, 627, 224

Gray, D. F. 1994, PASP, 106, 1248

Groenewegen, M. A. T. 2004, MNRAS, 353, 903

Groenewegen, M. A. T. 2007, A\&A, 474, 975

Groenewegen, M. A. T. 2008, A\&A, 488, 25

Holmberg, J., Nordström, B., \& Andersen, J. 2007, A\&A, 475, 519

Kanbur, S., Marconi, M., Ngeow, C., et al. 2009, in Stellar Pulsation: Challenges for Theory and Observation, ed. J. A. Guzik, \& P. A. Bradley, AIP Conf. Ser., 1170,18

Kervella, P., Bersier, D., Mourard, D., et al. 2004a, A\&A, 428, 587

Kervella, P., Nardetto, N., Bersier, D., Mourard, D., \& Coudé du Foresto, V. 2004b, A\&A, 416, 941

Kervella, P., Mérand, A., Szabados, L., et al. 2008, A\&A, 480, 167

Kovtyukh, V. V., \& Gorlova, N. I. 2000, A\&A, 358, 587

Kovtyukh, V. V., Andrievsky, S. M., Belik, S. I., \& Luck, R. E. 2005, AJ, 129, 433

Krockenberger, M., Sasselov, D., Noyes, R., et al. 1998, in Cool Stars, Stellar Systems, and the Sun, ed. R. A. Donahue, \& J. A. Bookbinder, ASP Conf. Ser., 154, 791

Laney, C. D., \& Stobie, R. S. 1995, MNRAS, 274, 337

Lemasle, B., François, P., Bono, G., et al. 2007, A\&A, 467, 283

Lemasle, B., François, P., Piersimoni, A., et al. 2008, A\&A, 490, 613

Lub, J., \& Pel, J. W. 1975, in Multicolor Photometry and the Theoretical HR Diagram, IAU Symp., 190, 133

Lub, J., \& Pel, J. W. 1977, A\&A, 54, 137

Luck, R. E., \& Andrievsky, S. M. 2004, AJ, 128, 343

Maciel, W. J., \& Costa, R. D. D. 2010, IAU Symp., 265, 317

Macri, L. M., Stanek, K. Z., Bersier, D., Greenhill, L. J., \& Reid, M. J. 2006, ApJ, 652, 1133
Marconi, M. 2009, Mem. Soc. Astron. Ital., 80, 141

Marengo, M., Karovska, M., Sasselov, D. D., et al. 2003, ApJ, 589, 968

Marengo, M., Evans, N. R., Barmby, P., Bono, G., \& Welch, D. 2009, in The Evolving ISM in the Milky Way and Nearby Galaxies

Mérand, A., Kervella, P., Coudé Du Foresto, V., et al. 2005, A\&A, 438, L9

Moskalik, P., Krzyt, T., Gorynya, N. A., \& Samus, N. N. 2000, in The Impact of Large-Scale Surveys on Pulsating Star Research, IAU Colloq. 176, ed. L. Szabados, \& D. Kurtz, ASP Conf. Ser., 203, 233

Nardetto, N., Fokin, A., Mourard, D., et al. 2004, A\&A, 428, 131

Nardetto, N., Mourard, D., Mathias, P., Fokin, A., \& Gillet, D. 2007, A\&A, 471, 661

Nardetto, N., Gieren, W., Kervella, P., et al. 2009, A\&A, 502, 951

Payne-Gaposchkin, C. 1951, Astrophysics: A Topical Symposium (New York: McGraw Hill), 495

Payne-Gaposchkin, C. 1954, Annals of Harvard College Observatory, 113, 153

Pedicelli, S., Lub, J., Pel, J. W., et al. 2008, Mem. Soc. Astron. Ital., 79, 539

Pedicelli, S., Bono, G., Lemasle, B., et al. 2009, A\&A, 504, 81

Pel, J. W. 1976, A\&AS, 24, 413

Pel, J. W., \& Lub, J. 1978, in The HR Diagram - The 100th Anniversary of Henry Norris Russell, ed. A. G. D. Philip, \& D. S. Hayes, IAU Symp., 80, 229

Petroni, S., Bono, G., Marconi, M., \& Stellingwerf, R. F. 2003, ApJ, 599, 522

Romaniello, M., Primas, F., Mottini, M., et al. 2008, A\&A, 488, 731

Sandage, A., Tammann, G. A., \& Reindl, B. 2009, A\&A, 493, 471

Scowcroft, V., Bersier, D., Mould, J. R., \& Wood, P. R. 2009, MNRAS, 396, 1287

Storm, J., Carney, B. W., Gieren, W. P., et al. 2004, A\&A, 415, 531

Sziládi, K., Vinkó, J., Poretti, E., Szabados, L., \& Kun, M. 2007, A\&A, 473, 579

Tammann, G. A., Sandage, A., \& Reindl, B. 2003, A\&A, 404, 423

van Leeuwen, F. 2007, A\&A, 474, 653

van Leeuwen, F., Feast, M. W., Whitelock, P. A., \& Laney, C. D. 2007, MNRAS, 379,723

van Loon, J. T., Gilmore, G. F., Omont, A., et al. 2003, MNRAS, 338, 857

Walraven, J. H., Tinbergen, J., \& Walraven, T. 1964, Bull. Astron. Inst. Netherlands, 17, 520

Welch, D. L., Alcock, C., Allsman, R. A., et al. 1997, in Variables Stars and the Astrophysical Returns of the Microlensing Surveys, ed. R. Ferlet, J.-P. Maillard, \& B. Raban, 205

Wesselink, A. J. 1946, Bull. Astron. Inst. Netherlands, 10, 91

Yin, J., Hou, J. L., Prantzos, N., et al. 2009, A\&A, 505, 497

Zoccali, M., Lecureur, A., Barbuy, B., et al. 2006, A\&A, 457, L1 